\title{
Quantitative Surface Analysis of a Binary Drug Mixture-Suppression Effects in the Detection of Sputtered Ions and Post-Ionized Neutrals
}

\author{
Gabriel Karras, ${ }^{1,2}$ Nicholas P. Lockyer ${ }^{1}$ \\ ${ }^{1}$ Photon Science Institute, School of Chemistry, University of Manchester, Oxford Rd., Manchester, M13 9PL, UK \\ ${ }^{2}$ University of Bourgogne, Dijon, France
}

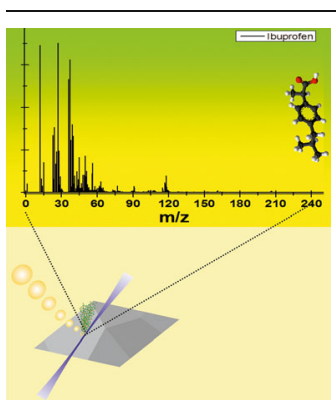

Abstract. A systematic mass spectrometric study of two of the most common analgesic drugs, paracetamol and ibuprofen, is reported. The drugs were studied by means of secondary ion mass spectrometry (SIMS) and secondary neutral mass spectrometry (SNMS) using laser post-ionization (LPI) both in pure samples and in a two-component mixture. Ion suppression within the two-component system observed in SIMS mode is ameliorated using LPI under room temperature analysis. However, suppression effects are apparent in LPI mode on performing the analysis at cryogenic temperatures, which we attribute to changes in the desorption characteristics of sputtered molecules, which influences the subsequent post-ionization efficiency. This suggests different mechanisms of ion suppression in SIMS and LPI modes.

Key words: Suppression effects, SIMS, SNMS, Laser post ionization, Paracetamol, Ibuprofen, Drug mixture

Received: 28 September 2013/Revised: 28 January 2014/Accepted: 29 January 2014

\section{Introduction}

N econdary ion mass spectrometry (SIMS) is a widely $\checkmark$ used technique applied to both fundamental and applied research. Biomedical applications and study of the chemistry taking place inside cells and tissues, surface analysis of polymers and semiconductor devices, and investigation of the composition of meteorites are just a few of the scientific areas where SIMS has been proven to be a valuable analytical tool [1-9].The main concept of the SIMS technique is to study the ionized particles (atoms, molecules, and clusters) emitted from a surface after the impact of a primary ion beam. However, sputtering, besides leading to the ejection of ionized particles, also results in the ejection of neutral species, which typically consist more than $90 \%$ of the sputtered material [10]. This predominance of neutral species is thought to be common in other stimulated desorption mass spectrometry techniques, including matrix assisted laser desorption ionization (MALDI), where for UV desorption, ionized fractions $10^{-3}$ to $10^{-7}$ are reported [11-13].

Electronic supplementary material The online version of this article (doi:10.1007/s13361-014-0847-6) contains supplementary material, which is available to authorized users.

Correspondence to: Gabriel Karras; e-mail: Gabriel.Karras@u-bourgogne.fr
Probing this neutral ejected material is achieved through various means of post-ionization using for example photons [14, 15], electrons [16], or an electrospray [17]. In molecular SIMS analysis, post-ionization (also known as secondary neutral mass spectrometry) is generally achieved through laser post-ionization (LPI). Up to now the technique has been used in a wealth of experiments, including the detection of trace elements, polyaromatic hydrocarbons, molecules in tissues and cells [18-20], the depth profiling of thin films [21], and fundamental studies of the sputtering process [22] to name a few.

In the current manuscript, we present results from the quantitative study of two widely used drugs, paracetamol and ibuprofen, by means of SIMS and LPI techniques.

Initially, the drugs were studied individually so as to probe the different information carried by the ionized and the postionized neutral sputtered material; then our measurements were extended to a binary mixture of the two analytes to assess quantification. Previous SIMS studies have demonstrated the ionization suppression of a molecular constituent when it is embedded in a complex environment [23, 24], a manifestation of a well-known effect in desorption mass spectrometry [25]. Here, we extend these investigations to secondary neutrals and consider both relative ion yield and the extent of fragmentation.

In the SIMS analysis of samples of biological origin, indepth molecular profiling studies, and in LPI experiments, cryogenic conditions are often used to retain a hydrated 
sample matrix, to improve depth resolution [26], or to reduce the contribution of photoionized gas phase species [27]. We, therefore, have studied the dependence of the sputtered material (both secondary ions and neutrals) on the temperature of the sample from a quantitative view point. In SIMS, the effects of analyzing samples in cryogenic and room temperature conditions have been studied previously by several groups [28-31], and it has been shown that there is a substantial increase in the detection of the protonated ions under frozen hydrated conditions. In our case, the samples were dehydrated prior to cooling by loading them into the vacuum system at room temperature.

\section{Experimental}

The molecules under study, paracetamol (acetaminophen) and ibuprofen, were purchased from Sigma-Aldrich, UK and used without any further purification. Analytes were dissolved in methanol to a concentration $10^{-3} \mathrm{M}$ and multilayer thin films were produced on $5 \times 5 \mathrm{~mm}^{2}$ silicon substrates using a spin caster (model WS-400BX6NPP.LITE; Laurell Technologies Corp. North Wales, PA, USA). Mixed samples were prepared by the same technique using a mixed solution with a concentration ratio ibuprofen:paracetamol of 10:6.

All the measurements presented here were conducted in a BioToF-SIMS instrument (Kore Technology Ltd., Ely, UK) described previously [32]. The sample substrates were mounted on a copper stage, which can be cooled to $\sim 150 \mathrm{~K}$ using the flow of nitrogen gas through a vessel containing liquid nitrogen and through the base of the stage. Sputtering was induced by the impact of an ion beam delivered by a gold cluster liquid metal ion source (Ionoptika Ltd., Southampton, UK). Owing to the relatively low molecular mass of the analytes, the primary ions chosen in the present study were $20 \mathrm{keV} \mathrm{Au}^{+}$, with a DC-equivalent current of $5 \mathrm{nA}$ onto the target, scanned over a target area $400 \times 400 \mu \mathrm{m}^{2}$. For the SIMS analysis, the duration of the ion beam pulse was $90 \mathrm{~ns}$, with delayed secondary ion extraction pulsing resulting in peak widths $\sim 8 \mathrm{~ns}$ (FWHM). All analyses were performed in the static-SIMS regime and the ion-fluences used were of the order of $10^{10}$ ions $/ \mathrm{cm}^{2}$. Sputtered ions were collected using an extraction optic placed $1 \mathrm{~cm}$ above the stage and analyzed in a dual stage ToF-reflectron mass spectrometer. Mass spectra were collected using a multichannel plate-photomultiplier detector (SAI Ltd., Manchester, UK), which was interfaced to a 2 ns transient digitizer (PDA500; Signatec Ltd., Lockport, Illinois, USA). The base pressure during the measurements was $<10^{-8}$ mbar.

Neutral sputtered species were post-ionized using a pulsed Nd:YAG laser source (model SL 312; EKSPLA Ltd., Vilnius, Lithuania). The laser system could deliver linearly polarized pulses with duration $150 \mathrm{ps}$ at four different wavelengths, 1064, 532, 355, and $266 \mathrm{~nm}$ with maximum energy per pulse $270,130,60$, and $40 \mathrm{~mJ}$, respectively, at a repetition rate of $10 \mathrm{~Hz}$. In the present communication, the recorded mass spectra were obtained using only the fourth harmonic at $266 \mathrm{~nm}$. The laser beam was focused in the vacuum chamber using a $\mathrm{MgF}_{2}$ lens with nominal focal length $25 \mathrm{~cm}$, producing a focused laser intensity of the order $10^{11} \mathrm{~W} / \mathrm{cm}^{2} \sim 1 \mathrm{~mm}$ above the sample. Temporal overlap of the laser pulse and the neutral plume pulse was achieved using a pulse delay generator (BNC 555; Berkley Nucleonics Corp., San Rafael, CA, USA) by tuning the triggering of the ion beam with respect to the arrival of the laser pulse in the interaction region. For the laser postionization measurements signal optimization required also the increase of the duration of the ion beam pulse to $1600 \mathrm{~ns}$. This provided increased data rates without compromising the mass resolution due to the decoupling of (post-) ionization and sputtering, and compensated for the low repetition rate of the laser used in this work. To avoid contribution from the sputtered ions (SIMS signal) to the LPI experiment, a suppression voltage of $-1.5 \mathrm{kV}$ is applied to the stage during the primary ion beam impact and the reflectron voltages optimized to transmit only the post-ionized neutral species. In addition, each LPI spectrum was corrected for the contribution of the background photoionization signal due to the residual gas in the vacuum chamber and the vapour pressure of the sample in a similar way as in [20] and described in detail below. To minimize this contribution, most of our LPI measurements were taken under cryogenic conditions, employing the above-mentioned setup. Both SIMS and LPI data were recorded in positive ion mode using identical detector settings to allow quantitative comparison.

\section{Results and Discussion}

Initially, we present and discuss the results obtained from pure samples using SIMS and LPI techniques to assess the relative ionization efficiency and fragmentation levels in each case.

\section{Analysis of Single Component Drug Samples}

In Fig. 1 typical mass spectra obtained from paracetamol in SIMS and LPI modes are presented. Under SIMS analysis, protonated molecular ions are observed for both paracetamol (Fig. 1a) and ibuprofen (not shown) at $\mathrm{m} / z 152$ and 207, respectively. Under LPI analysis, parent radical molecular ions for paracetamol (Fig. 1b) and ibuprofen (not shown) are recorded. The corresponding ibuprofen spectra are depicted in Figure S3 in the Supplementary Material.

In these studies, the analytes were prepared as thin films and held under cryogenic conditions to reduce the gas-phase concentration above the sample. In all cases, LPI spectra are background-corrected, meaning that the signal corresponding to any residual photoionized gas-phase species has been subtracted from the original spectrum, leaving only the contribution from sputtered neutrals that have been postionized. In addition, even though the repetition rate of the 


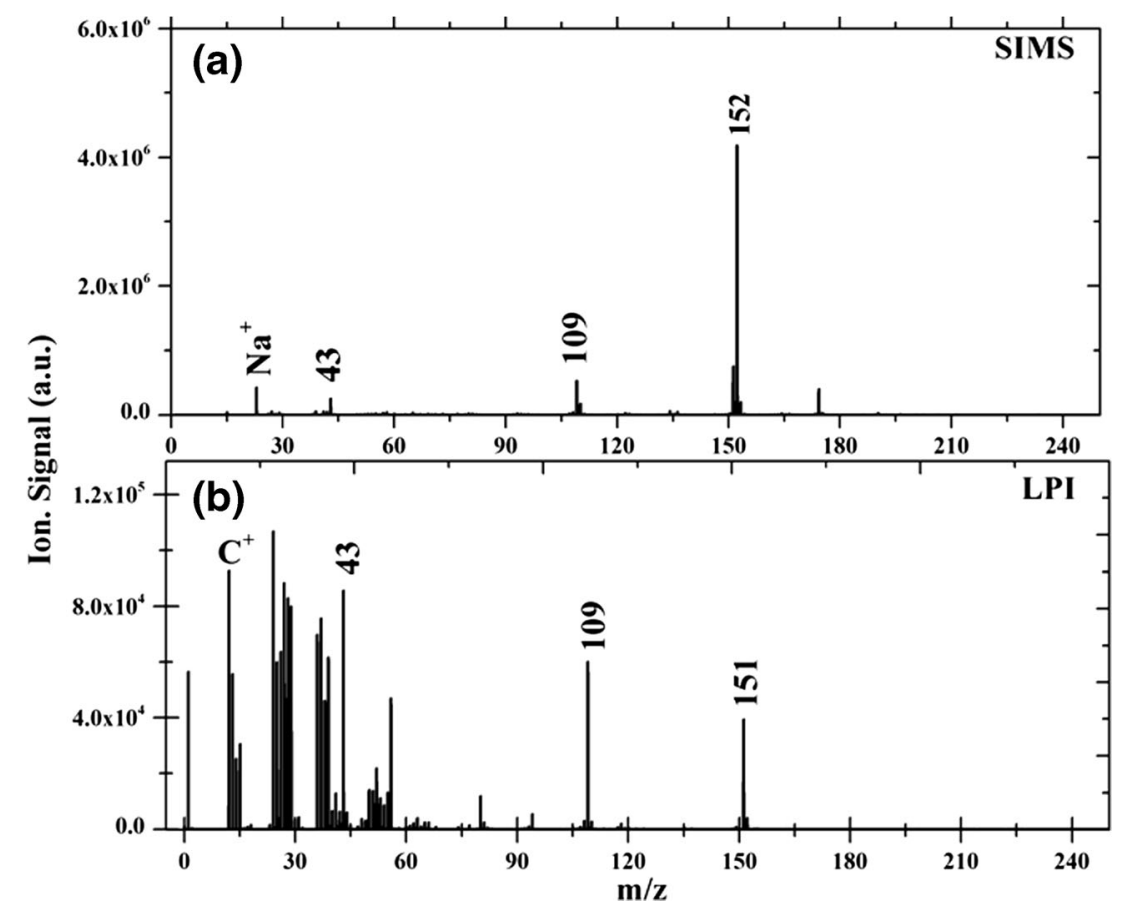

Figure 1. SIMS (a) and LPI (b) spectra of paracetamol. The primary ion dose was $5 \times 10^{10}$ ions $/ \mathrm{cm}^{2}$. Laser wavelength was $266 \mathrm{~nm}$ and the laser intensity at $5 \times 10^{11} \mathrm{~W} / \mathrm{cm}^{2}$

post-ionization experiment is much lower than in SIMS, the spectral dose on the sample was kept constant $\left(\sim 5 \times 10^{10}\right.$ ions $\left./ \mathrm{cm}^{2}\right)$ to allow a quantitative comparison of the relative signal levels between SIMS and LPI experiments. For both samples, the post-ionization laser intensity was $\sim 5 \times 10^{11} \mathrm{~W} / \mathrm{cm}^{2}$.

Figure 2 and Table 1 summarize the relative ion yields and degree of fragmentation of each molecule obtained under room temperature and cryogenic conditions using both SIMS and LPI methods. Under each condition, the ion yields are normalized to the molecular ion $(\mathrm{M}+\mathrm{H})$ or $\mathrm{M}$, respectively. The ratio of the molecular ion to the main diagnostic fragment in each case, $\left[\mathrm{M}-\mathrm{CH}_{3} \mathrm{CO}\right]^{+}(\mathrm{m} / \mathrm{z}$ 109) for paracetamol and $[\mathrm{M}-\mathrm{COOH}]^{+} \mathrm{m} / \mathrm{z} 161$ for ibuprofen, is generally higher for paracetamol than for ibuprofen indicating a case-sensitive fragmentation rate for each molecule under $\mathrm{Au}^{+}$ion beam bombardment. The exception to this is the room temperature LPI analysis, where the ratio is very similar for both molecules (Table 1).

For paracetamol, the production of protonated molecular ions is favored over the diagnostic fragment $m / z 109$ in SIMS analysis, whereas the corresponding ratio is reduced by an order of magnitude in the LPI analysis. This could reflect the relative stability of the paracetamol $(\mathrm{M}+\mathrm{H})$ ion, or that the $m / z$ 109 fragment is easily fragmented further under picosecond UV laser irradiation. In contrast, the extent of fragmentation of (protonated) molecular ion to $\mathrm{m} / z 161$ in ibuprofen is greater in SIMS compared with LPI (Table 1). This could be explained by the relative basicity values of the two analytes (see below). In these experiments, the sample temperature does not significantly affect the fragmentation behavior.
For both molecules, contrary to the mass spectra obtained in SIMS mode, in the case of the LPI measurements we observe extended fragmentation towards the low mass range, with significant production of $\mathrm{C}_{1-3}$ hydrocarbons. This behavior is expected and we attribute it to the long molecular chain of the molecules under study and also to the relatively long duration of the laser pulse. The diagnostic fragments considered in these sets of experiments contain a chromophoric group (the benzene ring) for the applied wavelength of $266 \mathrm{~nm}$, so subsequent photon absorption within the relatively long laser pulse is of high probability. Application of sub-picosecond and/or IR laser pulses may overcome this limitation [20, 33-35].

In order to gain further insight into the ionizationfragmentation mechanism of the molecules under study, the dependence of the detected photo-ion signal of several fragments on the laser power was examined. This dependence is presented in Figure S4 in the Supplementary Material for the parent and the main molecular fragment ions for paracetamol molecule. The ionization scheme employed in the present case is most probably that of multi-photon absorption as the characteristic slope in the $\log -\log$ plot is less than unity $(0.7 \pm 0.02)$ for both ions.

We consider next the relative sensitivity of SIMS and LPI measurements on these analytes under room temperature and cryogenic conditions. In Table 2, useful yields (the number of detected secondary species per primary ion impact) for each $m / z$ value are given for both analytes in SIMS and LPI experiments. Clearly, the absolute useful yield values will depend on the surface coverage of each analyte and, for this 


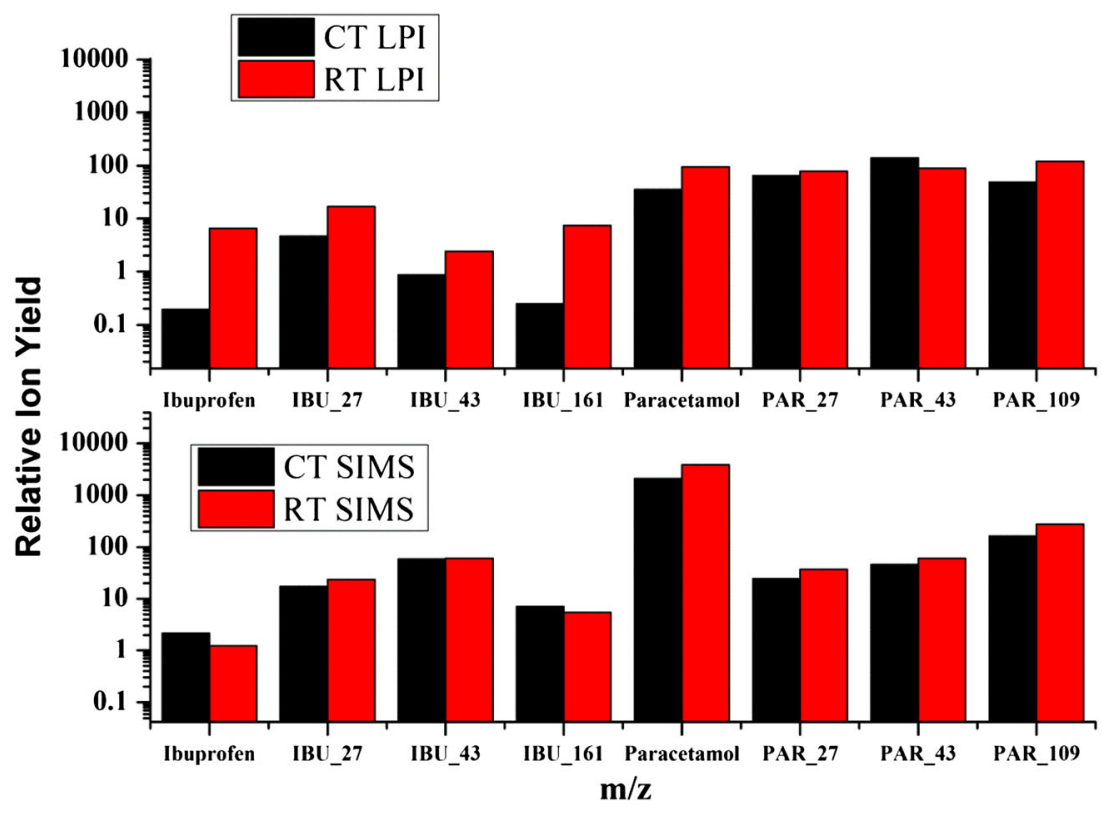

Figure 2. Relative ion yields recorded from paracetamol and ibuprofen samples at room temperature (RT) and cryogenic temperature (CT) in LPI (top) and SIMS (bottom) modes. (In the horizontal axis, fragments with same $m / z$ for both samples are noted using the first three letters of the parent molecule and then the $\mathrm{m} / \mathrm{z}$ of each fragment). As we see, the most significant dependence on the sample temperature is observed by the ibuprofen signals under LPI analysis

reason, the same area of each sample was analyzed using both SIMS and LPI modes to allow a direct comparison of useful yields and fragmentation. Table 2 contains the relative ion yields for SIMS and LPI obtained from the pure samples under room temperature and cryogenic conditions. Data for both molecular ion ' $\mathrm{M}$ ' (protonated for SIMS, radical for LPI) and the sum of the molecular ion and diagnostic fragment ' $\mathrm{M}+\mathrm{F}$ ' are given. Considering that the same areas of each molecular film are analyzed in both SIMS and LPI modes, we observe that under cryogenic conditions the SIMS yields are an order of magnitude higher than the LPI yields, both for molecular ions and diagnostic fragments. This may in part be due to LPI fragmentation into nondiagnostic ion channels, as indicated in Fig. 1b. Under room temperature analysis, the relative yield (for SIMS compared with LPI) for paracetamol is relatively unchanged, whereas for ibuprofen, the LPI yields now exceed the SIMS yields both for $\mathrm{M}$ and $\mathrm{M}+\mathrm{F}$ signals. As we are still correcting for gas-phase contributions in the LPI analysis, this observation suggests changes in the sputter characteristics of neutrals, which selectively enhance the LPI signal relative to the SIMS signal when analyzing the sample at room temperature. This is discussed further below.

In a separate study of bulk crystalline samples of paracetamol and ibuprofen, absolute SIMS useful yields were determined both for $(\mathrm{M}+\mathrm{H})$ and $\mathrm{F}$. The measured $(\mathrm{M}+\mathrm{H})$ yields of paracetamol and ibuprofen were $4 \times 10^{-4}$ and $5 \times 10^{-5}$, respectively, whereas the $\mathrm{F}$ yields were $8 \times 10^{-5}$ and $3 \times 10^{-4}$, respectively. Therefore, the combined SIMS yield of parent and diagnostic fragment ion for each analyte in a pure, bulk state is within a factor of two, although it should be noted that the $k_{\mathrm{a}}$ values of paracetamol and ibuprofen are 9.38 and 4.91, respectively [36]. These data are consistent with our combined SIMS/LPI measurements on thin films, where the $(\mathrm{M}+\mathrm{H}) / \mathrm{M}$ yield ratio is greater for paracetamol than for ibuprofen (Table 2). The more basic molecule (paracetamol), analyzed in isolation, shows an increased tendency to form protonated ions in the SIMS mode over radicals by photoionization in the LPI mode. It should be noted that in the thin film measurements, the ion detector gain was operated at a constant low value to avoid saturation effects from the photoionization of gas-phase species, whilst at the same time allowing quantitative comparisons of ion yield between SIMS and LPI measurement of different samples.

\section{Analysis of a Two-Component Drug Sample}

In this section we present and discuss the results obtained using SIMS and LPI techniques in a mixed sample

Table 1. Comparison of the Extent of Fragmentation in SIMS and LPI Analysis of Pure Samples of Paracetamol and Ibuprofen Under Room Temperature and Cryogenic Conditions. Diagnostic Fragments (F) at $m / z$ 109 and 161 from Paracetamol and Ibuprofen, Respectively, are Considered. SIMS and LPI Analyses Were Performed on the Same Area of Each Sample Under a Given Temperature, Using the Same Primary Ion Dose

\begin{tabular}{lllll}
\hline Fragmentation ratio & \multicolumn{2}{l}{ Cryogenic temperature } & \multicolumn{2}{l}{ Room temperature } \\
\cline { 2 - 5 } & $\begin{array}{lllll}\text { SIMS } \\
(\mathrm{M}+\mathrm{H}) / \mathrm{F}\end{array}$ & $\begin{array}{l}\text { LPI } \\
\mathrm{M} / \mathrm{F}\end{array}$ & $\begin{array}{l}\text { SIMS } \\
(\mathrm{M}+\mathrm{H}) / \mathrm{F}\end{array}$ & $\begin{array}{l}\text { LPI } \\
\mathrm{M} / \mathrm{F}\end{array}$ \\
\hline Paracetamol & 13 & 0.72 & 14 & 0.78 \\
Ibuprofen & 0.31 & 1.0 & 0.22 & 0.88
\end{tabular}


Table 2. Comparison of Relative Yields from SIMS and LPI Analysis of Pure Samples of Paracetamol and Ibuprofen Under Room Temperature and Cryogenic Conditions. The Yields of (Protonated) Molecular Ions and Diagnostic Fragments ( $\mathrm{m} / \mathrm{z} 109$ and 161) Are Considered. SIMS and LPI Analyses Were Performed on the Same Area of Each Sample Under a Given Temperature

\begin{tabular}{lllll}
\hline Relative yield SIMS/LPI & Cryogenic temperature & \multicolumn{2}{c}{ Room temperature } \\
\cline { 2 - 5 } & Paracetamol & Ibuprofen & Paracetamol & Ibuprofen \\
\hline$(\mathrm{M}+\mathrm{H}) / \mathrm{M}$ & 59 & 12 & 41 & 0.18 \\
{$[(\mathrm{M}+\mathrm{H})+\mathrm{F}] /(\mathrm{M}+\mathrm{F})$} & 26 & 28 & 19 & 0.47 \\
\hline
\end{tabular}

containing the two drugs, paracetamol and ibuprofen in the molar ratio 6:10, to assess ion suppression effects. Figure 3 presents typical mass spectra obtained from this sample using SIMS and LPI techniques. In both cases, the primary ion dose used was $\sim 6 \times 10^{10}$ ions $/ \mathrm{cm}^{2}$. For the LPI measurements, the laser intensity in the interaction region is estimated to be $\sim 5 \times 10^{11} \mathrm{~W} / \mathrm{cm}^{2}$.

The relative ion yields for each drug in the twocomponent mixture under various analysis conditions are shown in Table 3. The relative ion yields have been corrected for the expected surface concentrations given the original 6:10 molar ratio in the mixed sample solution. We have assumed that no surface segregation occurs, and to avoid any influence of lateral segregation, we have ensured that consistent areas were analyzed in both SIMS and LPI modes. On this basis, paracetamol exhibits a much greater ion yield, both in SIMS and LPI experiments under cryogenic conditions compared with ibuprofen. SIMS analysis provides a very similar picture under room temperature condition. In the SIMS analyses, the protonated molecular ion ratio $(\mathrm{M}+\mathrm{H})_{\mathrm{para}} /(\mathrm{M}+\mathrm{H})_{\text {ibup }}$ is $\sim 400$. The ratio is reduced by a factor of 10 when also considering the major fragment ions, which are not themselves protonated. This is in contrast to the SIMS yield ratios (paracetamol/ibuprofen) from these analytes in pure bulk form, which were determined to be $\sim 10$ for $(\mathrm{M}+\mathrm{H})$ and $\sim 1$ including also $\mathrm{F}$ (the diagnostic fragment ions) as reported above. At this point, it seems that we are dealing with an ionization suppression effect in excess of one order of magnitude. In the context of biomolecular, room temperature SIMS analysis, this has previously been described by Jones et al. $[23,24]$, who attributed the observed behavior to the gas phase basicity of the constituent analytes. As mentioned above, the $\mathrm{p} k_{\mathrm{a}}$ values of paracetamol and ibuprofen are 9.38
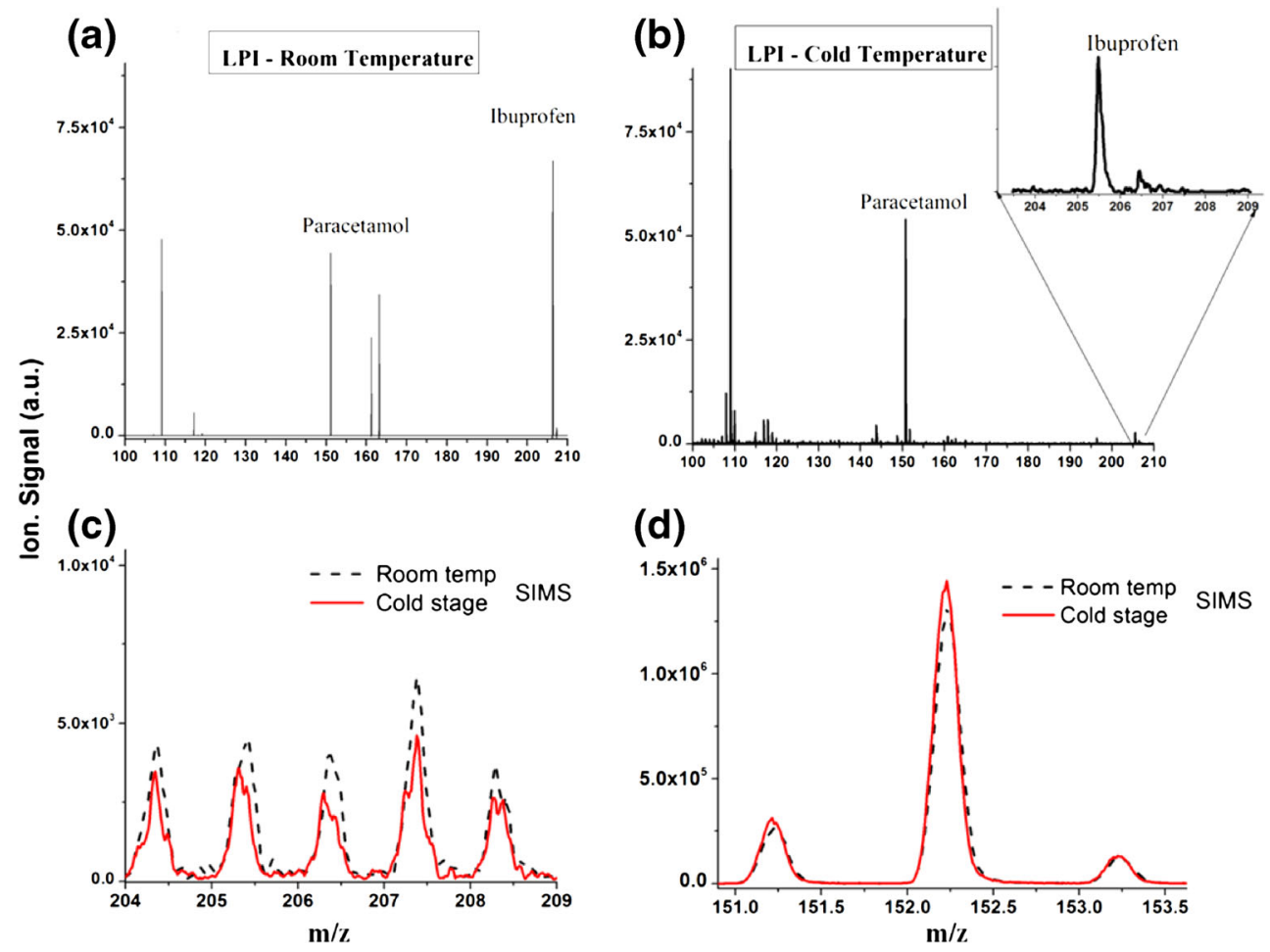

Figure 3. Mass spectra of a two-component drug mixture acquired using (a) LPI at room temperature, (b) LPI at cryogenic conditions. Panels (c) and (d) show SIMS data overlayed at each temperature for the molecular ion region of ibuprofen and paracetamol, respectively. For LPI laser wavelength was $266 \mathrm{~nm}$ with intensity $5 \times 10^{11} \mathrm{~W} / \mathrm{cm}^{2}$. The primary ion dose was $5 \times$ $10^{10}$ ions $/ \mathrm{cm}^{2}$ and the rastered area $400 \times 400 \mu \mathrm{m}^{2}$ in all analyses 
Table 3. Comparison of Relative Ion Yields Under SIMS and LPI Analyses for a Mixed Sample Containing Paracetamol and Ibuprofen in the Ratio 6:10. The Sample Was Analyzed under Cryogenic and Room Temperature Conditions and Ratios of (Protonated) Molecular Ion Signals (Both Given the Symbol M for Clarity) with and without the Contribution of Diagnostic Fragment Ions (F) at $m / z 109$ and 161 Included. Ion Yields Have Been Normalized to the Original Concentration Ratio

\begin{tabular}{lllll}
\hline $\begin{array}{l}\text { Relative yield } \\
\text { paracetamol /ibuprofen }\end{array}$ & \multicolumn{2}{l}{ Cryogenic temperature } & \multicolumn{2}{l}{ Room temperature } \\
\cline { 2 - 5 } & SIMS & LPI & SIMS & LPI \\
\hline $\mathrm{M}_{\text {para }} / \mathrm{M}_{\text {ibup }}$ & 440 & 26 & 390 & 1.4 \\
$(\mathrm{M}+\mathrm{F})_{\text {para }} /(\mathrm{M}+\mathrm{F})_{\text {ibup }}$ & 36 & 36 & 32 & 1.1 \\
\hline
\end{tabular}

and 4.91, respectively. In a two-component mixture, therefore, where competition for protons may exist, paracetamol is more likely to form protonated molecular ions in SIMS mode. This is what we observe in the ratios $\mathrm{M}_{\text {para }}$ / $\mathrm{M}_{\text {ibup }}$ and $(\mathrm{M}+\mathrm{F})_{\text {para }} /(\mathrm{M}+\mathrm{F})_{\text {ibup }}$ shown in Table 3 .

The LPI result at room temperature is significantly different, with a relative ion yield close to unity for both the radical molecular ions and $(\mathrm{M}+\mathrm{F})$ signals (Table 3, final column). It is immediately obvious from the mass spectra in Fig. $3 a$ and $b$ that the change in relative ion yield between SIMS and LPI modes is the result of a very significant increase in the LPI signals from ibuprofen $(\mathrm{m} / \mathrm{z} 207$ and 161) under room temperature conditions, whereas for paracetamol the $\mathrm{M}$ signal $(\mathrm{m} / \mathrm{z} 151)$ and the F signal $(\mathrm{m} / \mathrm{z} 109)$ show a relatively small temperature dependence under LPI. These observations are consistent with the LPI data from the single-component samples (Fig. 1), where the most significant change was again seen in the increased ibuprofen yields on going from cryogenic to room temperature analysis. It should be noted that as with the singlecomponent samples, these results are background-corrected for gas phase species.

The mechanism of this apparently enhanced LPI yield for ibuprofen is as yet unclear. Altered sputtering yield is thought to be unlikely since that would also affect the SIMS signal, as would any changes in surface concentration. In fact, the change of the SIMS signal attributable to the change of the stage temperature is negligible (Fig. 3c and d). It has been reported elsewhere that the temperature of the sample can play a significant role in the sputtered ionic signal $[26$, 37]. In our case, this was not observed, which can be

Table 4. Comparison of Extent of Fragmentation in SIMS and LPI Analyses of a Mixed Sample of Paracetamol and Ibuprofen (6:10 Molar Ratio) Under Room Temperature and Cryogenic Conditions. 'F' Denotes Diagnostic Fragments $m / z 109$ and 161 from Paracetamol and Ibuprofen, respectively

\begin{tabular}{lllll}
\hline Fragmentation ratio & \multicolumn{2}{l}{ Cryogenic temperature } & \multicolumn{2}{l}{ Room temperature } \\
\cline { 2 - 5 } & $\begin{array}{l}\text { SIMS } \\
(\mathrm{M}+\mathrm{H}) / \mathrm{F}\end{array}$ & $\begin{array}{l}\text { LPI } \\
\mathrm{M} / \mathrm{F}\end{array}$ & $\begin{array}{l}\text { SIMS } \\
(\mathrm{M}+\mathrm{H}) / \mathrm{F}\end{array}$ & $\begin{array}{l}\text { LPI } \\
\mathrm{M} / \mathrm{F}\end{array}$ \\
\hline $\begin{array}{l}\text { Paracetamol } \\
\text { Ibuprofen }\end{array}$ & 5.7 & 0.72 & 7.5 & 5.0 \\
\hline
\end{tabular}

attributed to our low-dose bombardment conditions and the fact that we were not observing molecular clusters, which appear to show a temperature effect even at low dose.

The fragmentation behavior of each analyte in the twocomponent drug mixture is shown in Table 4. For ibuprofen, the yield ratios $(\mathrm{M}+\mathrm{H}) / \mathrm{F}$ and $(\mathrm{M} / \mathrm{F})$ for SIMS and LPI analysis, respectively, show very little dependence on the sample temperature despite the very significant yield increase in LPI mode as noted above. For paracetamol, although the $(\mathrm{M}+\mathrm{H}) / \mathrm{F}$ ratio is consistent in SIMS mode, the $\mathrm{M} / \mathrm{F}$ ratio increases significantly (from 0.72 to 5.0 ) in LPI mode on going from cryogenic to room temperature conditions. Examining the corresponding absolute yields provides some insight into the cause of this effect. Under the low detector gain conditions of this particular analysis, the LPI molecular ion yield of paracetamol from the mixed sample remains at $1 \pm 0.2 \times 10^{-7}$, whereas the $\mathrm{m} / \mathrm{z} 109$ fragment yield decreases from $1 \times 10^{-7}$ in the cryogenic sample to $2 \times 10^{-8}$ in the room temperature analysis. The increased thermal energy (of the order meV) of the sputtered species at room temperature is insignificant compared with the expected molecular ionization potential $(>6 \mathrm{eV})$, and to the energy imparted by the photon absorption (4.7 eV per photon). One possibility is that the kinetic energy distribution of the sputtered paracetamol molecule and/or the neutral fragment at $109 \mathrm{u}$ differs in the mixed sample under cryogenic and room temperature conditions. This could lead to a change in the temporal overlap of sputtered neutral and the laser pulse for one or both species, resulting in a change in the observed ion ratio. This is discussed further in the following section. Interestingly, this altered fragmentation effect is not so significant in the pure paracetamol sample, where the $\mathrm{M} / \mathrm{F}$ ratio increases only by $\sim 10 \%$ in the room temperature analysis (Table 1), and is not observed in the ibuprofen data.

\section{Temperature-Dependent Post-Ionization of Sputtered Neutrals}

Another interesting feature that was observed regarding the temperature dependence of the LPI signal is demonstrated in Fig. 4, where different mass ranges for the background corrected mass spectra are presented for room temperature and cryogenic conditions.

There are two cases concerning the dependence of different ions on sample temperature. The first case concerns the species that are not affected by the change of the temperature. This is clearly demonstrated in the insets of Fig. 4, where we it is seen that background levels of atomic ions such as $\mathrm{Na}^{+}$and $\mathrm{Fe}^{+}$maintain their signal levels during the cooling process. The second case has to do with the significant changes that the LPI signal of the molecular fragments such as $m / z 77\left(\mathrm{C}_{6} \mathrm{H}_{5}\right)^{+}$and $m / z 91\left(\mathrm{C}_{7} \mathrm{H}_{7}\right)^{+}$ undergo during the cooling process. 

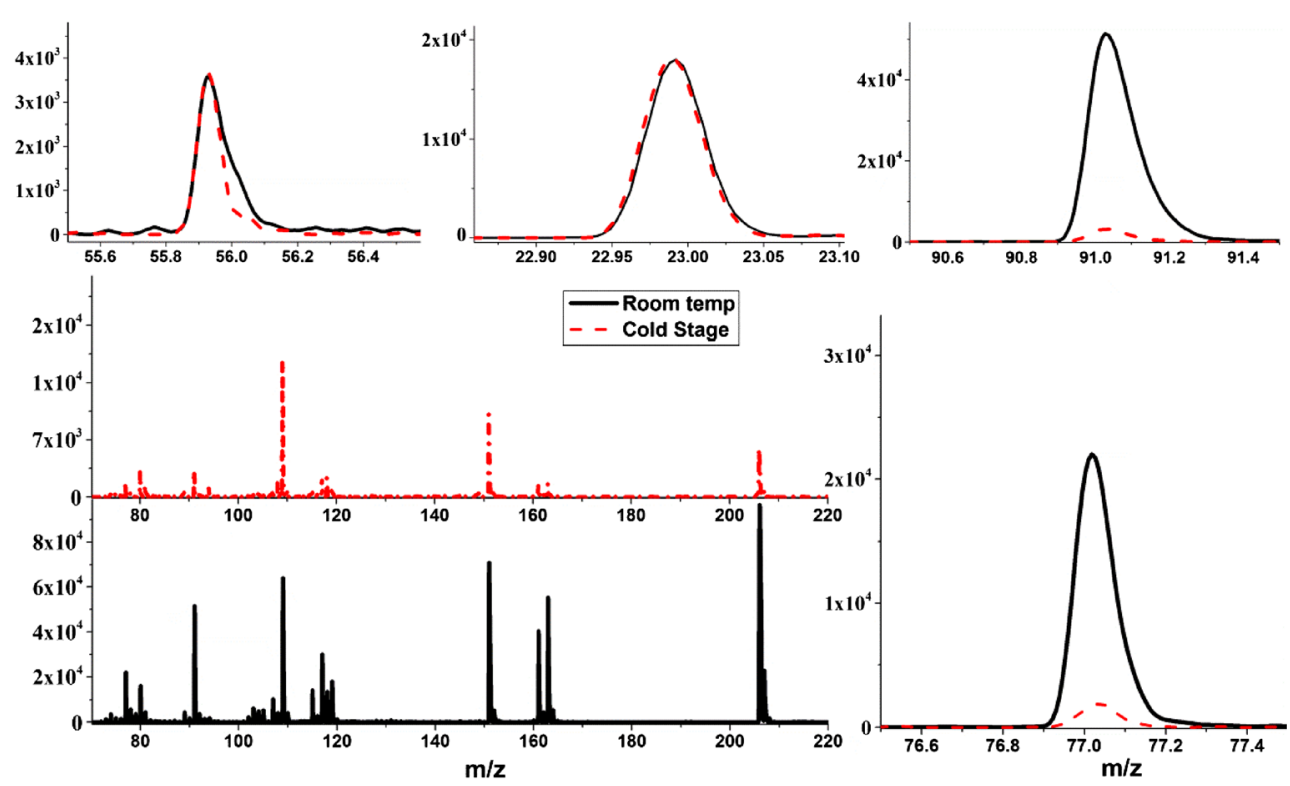

Figure 4. Expanded regions of the LPI mass spectra of the two-component drug sample showing signals that temperature sensitive and those that are not. Data recorded at room temperature (single line) and cryogenic conditions (dashed line) are overlayed

A dependence of molecular fragment emission on surface temperature and molecular mass has previously been reported for self-assembled organic monolayers (SAMs) under $\mathrm{keV} \mathrm{Ar}^{+}$ bombardment [38-40] and thick organic samples upon the impact of $\mathrm{C}_{60}{ }^{+}$projectiles [40]. In the case of SAM systems, in addition to a momentum transfer process (of $\mathrm{eV}$ magnitude) resulting directly from the collision cascade, a two-step mechanism was suggested. This two-step process involved first fragmentation of surface-bound molecules, which then evaporate with low energy $\left(\sim 10^{-2} \mathrm{eV}\right)$ from a physisorbed state, having reached thermal equilibrium with the surface. Surface cooling was reported to lead to a broader, lower energy distribution of emitted molecules [37]. Such a broadening would be consistent with a lower post-ionization probability for molecules sputtered from a room temperature sample, whereas the atomic neutrals resulting from the collision cascade would be unaffected. As highlighted by Postawa et al. [40], this molecular evaporative mechanism most likely applies in systems where the energy of the primary ion impact is rapidly dissipated into the substrate, such as thin films under atomic primary ion bombardment. Concerning the desorption dynamics found in thick organic films, Brenes et al. [41] suggest an evaporative mechanism induced by the localized energy deposition following a $\mathrm{C}_{60}{ }^{+}$ion impact on the surface results in the temperature-dependent formation of low mass fragments. The flight time distribution of these fragments was found to be wider and peak at a lower energy at a sample temperature $300 \mathrm{~K}$ in comparison to $100 \mathrm{~K}$. It is interesting to note that for these thick films under polyatomic bombardment the broader, lower peak energy distribution is observed at the higher sample temperature, whereas for the SAMs under atomic bombardment this was observed for the lower sample temperature [37].
In the same paper, Brenes et al. report that in comparison, the energy distribution of post-ionized intact molecules sputtered by $\mathrm{Au}^{+}$shows only a $0.01 \mathrm{eV}$ decrease in the most probable kinetic energy and 'slight differences' in the highenergy component on changing the sample temperature from 100 to $300 \mathrm{~K}$ (no data is presented for the fragments generated under $\mathrm{Au}^{+}$bombardment). The authors do not comment on the relative signal levels recorded at the two temperatures.

Further insight into the mechanism(s) behind the temperature effects observed in our experiments come from the evidence that they appear to be more significant for the LPI of ibuprofen than for paracetamol, both in single- and twocomponent samples. However, assuming a predominantly physisorption process, the binding energies and therefore the flight times to the post-ionization volume of the two molecules of similar size and chemistry are expected to be similar. This suggests the internal energy of sputtered species and their stability towards fragmentation following post-ionization may be important factors in the observed temperature dependence of the resulting mass spectra.

\section{Conclusion}

The analysis of a mixed sample of paracetamol and ibuprofen shows ion suppression effects in SIMS mode, which can complicate quantitative analysis. This is apparent particularly in the relative ion yields of protonated molecular ions and can be explained qualitatively in terms of relative gas phase basicity. This effect is overcome to a greater or lesser extent using LPI analysis of room temperature or cryogenic samples, respectively. Whilst the sample temperature has almost no influence of the SIMS spectra of these 
analytes, in LPI mode temperature-dependent changes in relative ion yields and the degree of fragmentation are observed depending on the analyte and its environment. Atomic LPI signals appear to be unaffected by this temperature dependence. This has implications for quantitative analysis and also for analyte identification based on matching fragment ion intensity in mixed samples. The mechanism behind the changes in LPI characteristics, which appears to be analyte- and sample-dependent, requires further study. Further energy-resolved experiments supported by molecular dynamic simulations are required before a definite conclusion can be reached regarding the origin of the observed LPI temperature dependence.

\section{Acknowledgments}

The authors acknowledge support for this work by the UK Biotechnology and Biological Sciences Research Council, BBSRC, under grant BB/I023771/1. The authors are grateful to John Vickerman and Adam McMahon for stimulating discussions.

\section{References}

1. Ninomiya, S., Ichiki, K., Yamada, H., Nakata, Y., Seki, T., Aoki, T., Matsuo, J.: Precise and fast secondary ion mass spectrometry depth profiling of polymer materials with large Ar cluster ion beams. Rapid Commun. Mass Spectrom. 23, 1601-1606 (2009)

2. Brunelle, A., Touboul, D., Laprévote, O.: Biological tissue imaging with time-of-flight secondary ion mass spectrometry and cluster ion sources. J. Mass Spectrom. 40, 985-999 (2005)

3. McDonnell, L.A., Piersma, S.R., Altelaar, A.F.M., Mize, T.H., Luxembourg, S.L., Verhaert, P.D., van Minnen, J., Heeren, R.M.A.: Subcellular imaging mass spectrometry of brain tissue. J. Mass Spectrom. 40, 160-168 (2005)

4. Bich, C., Havelund, R., Moellers, R., Touboul, D., Kollmer, F., Niehuis, E., Gilmore, I.S., Brunelle, A.: Argon cluster ion source evaluation on lipid standards and rat brain tissue samples. Anal. Chem. 85, 77457752 (2013)

5. Touboul, D., Halgand, F., Brunelle, A., Kersting, R., Tallarek, E., Hagenhoff, B., Laprévote, O.: Tissue molecular ion imaging by gold cluster ion bombardment. Anal. Chem. 76, 1550-1559 (2004)

6. Ostrowski, S.G., Van Bell, C.T., Winograd, N., Ewing, A.G.: Mass spectrometric imaging of highly curved membranes during tetrahymena mating. Science 305, 71-73 (2004)

7. Ravanel, X., Trouiller, C., Juhel, M., Wyon, C., Kwakman, L.F.T., Léonard, D.: Static time-of-flight secondary ion mass spectrometry analysis of microelectronics related substrates using a polyatomic ion source. Appl. Surf. Sci. 255, 1440-1442 (2008)

8. Wagner, M.S., Castner, D.G.: Characterization of adsorbed protein films by time-of-flight secondary ion mass spectrometry with principal component analysis. Langmuir 17, 4649-4660 (2001)

9. King, A.J., Henkel, T., Rost, D., Lyon, I.C.: Trace element depth profiles in presolar silicon carbide grains. Meteorit. Planet. Sci. 47, 1624-1643 (2012)

10. Benninghoven, A., Hagenhoff, B., Niehuis, E.: Surface MS: probing real-world samples. Anal. Chem. 65, 630A-640A (1993)

11. Ens, W., Mao, Y., Mayer, F., Standing, K.G.: Properties of matrixassisted laser desorption. Measurements with a time-to-digital converter. Rapid Commun. Mass Spectrom. 5, 117-123 (1991)

12. Mowry, C.D., Johnston, M.V.: Simultaneous detection of ions and neutrals produced by matrix-assisted laser desorption. Rapid Commun. Mass Spectrom. 7, 569-575 (1993)

13. Quist, A., Huth-Fehre, T., Sundqvist, B.: Total yield measurements in matrix-assisted laser-desorption using a quartz-crystal microbalance. Rapid Commun. Mass Spectrom. 8, 149-154 (1994)
14. Young, C.E., Pellin, M.J., Calaway, W.F., Jorgensen, B., Schweitzer, E.L., Gruen, D.M.: Trace surface analysis via RIS/TOF mass spectrometry. Nucl. Inst. Methods Phys. Res. B27, 119-129 (1987)

15. Ebata, S., Ishihara, M., Kumondai, K., Mibuka, R., Uchino, K., Yurimoto, H.: Development of an ultra-high performance multi-turn TOF-SIMS/SNMS system "MULTUM-SIMS/SNMS". J. Am. Soc. Mass Spectrom. 24, 222-229 (2013)

16. Oechsner, H.: Secondary neutral mass spectrometry (SNMS) -recent methodical progress and applications to fundamental studies in particle/ surface interaction. Int. J. Mass Spectrom. Ion Processes. 143, 271-282 (1995)

17. Nemes, P., Vertes, A.: Laser Ablation electrospray ionization for atmospheric pressure, in vivo, and imaging mass spectrometry. Anal. Chem. 79, 8098-8106 (2007)

18. Tyler, B.J., Dambach, S., Galla, S., Peterson, R.E., Arlinghaus, H.F.: Investigation of the utility of laser-secondary neutral mass spectrometry for the detection of polyaromatic hydrocarbons in individual atmospheric aerosol particles. Anal. Chem. 84, 76-82 (2012)

19. Haase, A., Arlinghaus, H.F., Tentschert, J., Jungnickel, H., Graf, P., Mantion, A., Draude, A., Galla, S., Plendl, J., Goetz, M.E., Masic, A., Meier, W., Thünemann, A.F., Taubert, A., Luch, A.: Application of laser post-ionization secondary neutral mass spectrometry/time-of-flight secondary ion mass spectrometry in nanotoxicology: visualization of nanosilver in human macrophages and cellular responses. ACS Nano 5, 3059-3068 (2011)

20. Willingham, D., Brenes, D.A., Wucher, A., Winograd, N.: Strong-field photoionization of sputtered neutral molecules for molecular depth profiling. J. Phys. Chem. C 114, 5391-5399 (2010)

21. Poerschke, D., Wucher, A.: Depth profiling of anodic tantalum oxide films with gold cluster ions. Surf. Interface Anal. 43, 171-174 (2011)

22. Zhou, J., Takahashi, L.K., Wilson, K.R., Leone, S.R., Ahmed, M. Internal energies of ion-sputtered neutral tryptophan and thymine molecules determined by vacuum ultraviolet photoionization. Anal. Chem. 82, 3905-3913 (2010)

23. Jones, E.A., Lockyer, N.P., Kordys, J., Vickerman, J.C.: Suppression and enhancement of secondary ion formation due to the chemical environment in static-secondary ion mass spectrometry. J. Am. Soc. Mass Spectrom. 18, 1559-1567 (2007)

24. Jones, E.A., Lockyer, N.P., Vickerman, J.C.: Suppression and enhancement of non-native molecules within biological systems. Appl. Surf. Sci. 252, 6727-6730 (2006)

25. Knochenmuss, R., Zenobi, R.: MALDI Ionization: in-plume processes. Chem. Rev. 103, 441-452 (2003)

26. Mao, D., Wucher, A., Brenes, D.A., Lu, C., Winograd, N.: Cluster secondary ion mass spectrometry and the temperature dependence of molecular depth profiles. Anal. Chem. 84, 3981-3989 (2012)

27. Wood, M., Zhou, Y., Brummel, C.L., Winograd, N.: Imaging with ion beams and laser postionization. Anal. Chem. 66, 2425-2432 (1994)

28. Piwowar, A.M., Fletcher, J.S., Kordys, J., Lockyer, N.P., Winograd, N., Vickerman, J.C.: Effects of cryogenic sample analysis on molecular depth profiles with TOF-secondary ion mass spectrometry. Anal. Chem. 82, 8291-8299 (2010)

29. Roddy, T.P., Cannon Jr., D.M., Ostrowski, S.G., Ewing, A.G., Winograd, N.: Proton transfer in time-of-flight secondary ion mass spectrometry studies of frozen-hydrated dipalmitoylphosphatidylcholine. Anal. Chem. 75, 4087-4094 (2003)

30. Mahoney, C.M., Fahey, A.J., Gillen, G., Xu, G., Batteas, J.D.: Temperature-controlled depth profiling in polymeric materials using cluster secondary ion mass spectrometry (SIMS). Appl. Surf. Sci. 252, 6502-6505 (2006)

31. Sjövall, P., Rading, D., Ray, S., Yang, L., Shard, A.G.: Sample cooling or rotation improves $\mathrm{C} 60$ organic depth profiles of multilayered reference samples: results from a VAMAS interlaboratory study. J. Phys. Chem. B 114, 769-774 (2010)

32. Braun, R.M., Blenkinsopp, P., Mullock, S.J., Corlett, C., Willey, K.F., Vickerman, J.C., Winograd, N.: Performance characteristics of a chemical imaging time-of-flight mass spectrometer. Rapid Commun. Mass Spectrom. 12, 1246-1252 (1998)

33. Szaflarski, D.M., El-Sayed, M.A.: Kinetic energy and formation mechanisms of iodine $(1+)$ and methyl(1+) from 266-nm picosecond versus nanosecond laser multiphoton absorption. J. Phys. Chem. 92, 2234-2239 (1988) 
G. Karras and N. P. Lockyer: Surface Analysis of a Binary Drug Mixture

34. Lockyer, N.P., Vickerman, J.C.: Multiphoton ionization mass spectrometry of small biomolecules with nanosecond and femtosecond laser pulses. Int. J. Mass Spectrom. 176, 77-86 (1998)

35. Longobardo, A., Macpherson, A.N., Vickerman, J.C., Lockyer, N.P.: New prospects for molecular post-ionisation using femtosecond IR lasers. Surf. Interface Anal. 45, 525-528 (2013)

36. Available at: www.drugbank.ca. Accessed June 28, 2013

37. Piwowar, A.M., Fletcher, J.S., Lockyer, N.P., Vickerman, J.C.: Investigating the effect of temperature on depth profiles of biological material using ToF-SIMS. Surf. Interface Anal. 43, 207-210 (2011)

38. Riederer, D.E., Chatterjee, R., Rosencrance, S.W., Postawa, Z., Dunbar, T.D., Allara, D.L., Winograd, N.: Thermal desorption induced by kilo-electronvolt ion bombardment of thiol-bound selfassembled monolayers on gold. J. Am. Chem. Soc. 119, 8089-8094 (1997)

39. Cyganik, P., Postawa, Z., Meserole, C.A., Vandeweert, E., Winograd, N.: Ion-induced erosion of organic self-assembled monolayers. Nucl. Inst. Methods Phys. Res. B 148, 137-142 (1999)

40. Postawa, Z., Meserole, C.A., Cyganik, P., Szymonska, J., Winograd, N.: Emission of neutral molecules from ion-bombarded thiol selfassembled monolayers. Nucl. Inst. Methods Phys. Res. B 182, 148-154 (2001)

41. Brenes, D.A., Willingham, D., Winograd, N., Postawa, Z.: Temperature effects in the sputtering of a molecular solid by energetic atomic and cluster projectiles. Surf. Interface Anal. 43, 78-80 (2011) 